\title{
Rule-Evolutionary Approach for Single-Stage Multi-Product Scheduling with Parallel Units
}

\author{
Yaohua He, Chi-Wai Hui \\ Chemical Engineering Department, Hong Kong University of Science and Technology, Clear \\ Water Bay, Hong Kong, P. R. China
}

\section{Supporting information}

Number pages: 10

Number of Tables: 8 


\section{Supporting Information}

\section{A. Data for Example 2}

Table A-1 order due date $\left(d_{j}\right)$ and order release time $\left(o r_{j}\right)$

\begin{tabular}{|c|c|c|c|c|c|c|c|c|c|c|c|c|c|c|c|c|c|}
\hline Order & $\mathrm{j} 1$ & $\mathrm{j} 2$ & j3 & $\mathrm{j} 4$ & $\mathrm{j} 5$ & j6 & j7 & j8 & j9 & $\mathrm{j} 10$ & $\mathrm{j} 11$ & $\mathrm{j} 12$ & j13 & j14 & $\mathrm{j} 15$ & $\mathrm{j} 16$ & $\mathrm{j} 17$ \\
\hline$d_{j}$ & 20 & 44 & 50 & 40 & 56 & 60 & 34 & 46 & 60 & 60 & 40 & 64 & 70 & 60 & 76 & 80 & 74 \\
\hline$o r_{j}$ & 0 & 0 & 0 & 0 & 0 & 0 & 0 & 0 & 0 & 0 & 0 & 0 & 0 & 0 & 0 & 0 & 0 \\
\hline Order & $\mathrm{j} 18$ & j19 & j20 & $\mathrm{j} 21$ & $\mathrm{j} 22$ & $\mathrm{j} 23$ & j24 & $\mathrm{j} 25$ & j26 & $\mathrm{j} 27$ & $\mathrm{j} 28$ & j29 & $\mathrm{j} 30$ & j31 & $\mathrm{j} 32$ & $\mathrm{j} 33$ & j34 \\
\hline$d_{j}$ & 66 & 80 & 80 & 20 & 44 & 50 & 40 & 56 & 60 & 34 & 46 & 60 & 60 & 40 & 64 & 70 & 60 \\
\hline$o r_{j}$ & 0 & 0 & 0 & 0 & 0 & 0 & 0 & 0 & 0 & 0 & 0 & 0 & 0 & 0 & 0 & 0 & 0 \\
\hline Order & $\mathrm{j} 35$ & j36 & j37 & j38 & j39 & $\mathrm{j} 40$ & $\mathrm{j} 41$ & $\mathrm{j} 42$ & $\mathrm{j} 43$ & j44 & $\mathrm{j} 45$ & $\mathrm{j} 46$ & $\mathrm{j} 47$ & $\mathrm{j} 48$ & $\mathrm{j} 49$ & $\mathrm{j} 50$ & \\
\hline$d_{j}$ & 76 & 80 & 74 & 66 & 80 & 80 & 20 & 44 & 50 & 40 & 56 & 60 & 34 & 46 & 60 & 60 & \\
\hline$o r_{j}$ & 0 & 0 & 0 & 0 & 0 & 0 & 0 & 0 & 0 & 0 & 0 & 0 & 0 & 0 & 0 & 0 & \\
\hline
\end{tabular}

Table A-2 Process time $\left(p_{j u}\right)$ and unit release time $\left(u r_{u}\right)$

\begin{tabular}{|c|c|c|c|c|c|c|c|c|c|c|c|c|c|c|}
\hline Order & u1 & $\mathbf{u} 2$ & $\mathbf{u} 3$ & u4 & Order & $\mathbf{u 1}$ & $\mathbf{u} 2$ & $\mathbf{u} 3$ & u4 & Order & u1 & $\mathbf{u} 2$ & u3 & $\mathrm{u} 4$ \\
\hline $\mathrm{j} 1$ & 10.20 & 3.60 & 4.20 & 10.80 & $\mathrm{j} 18$ & 6.00 & 7.25 & 5.00 & 9.50 & $\mathrm{j} 35$ & 5.70 & 4.44 & 5.70 & 4.17 \\
\hline $\mathrm{j} 2$ & 7.20 & 10.50 & 4.50 & 4.50 & $\mathrm{j} 19$ & 3.20 & 3.70 & 1.90 & 2.20 & $\mathrm{j} 36$ & 10.20 & 16.00 & 6.50 & 12.60 \\
\hline j3 & 6.25 & 5.20 & 5.50 & 5.00 & $\mathrm{j} 20$ & 3.00 & 4.80 & 5.00 & 5.20 & j37 & 4.55 & 17.50 & 5.50 & 5.25 \\
\hline $\mathrm{j} 4$ & 11.20 & 13.60 & 15.40 & 12.00 & $\mathrm{j} 21$ & 10.20 & 3.60 & 4.20 & 10.80 & $\mathrm{j} 38$ & 6.00 & 7.25 & 5.00 & 9.50 \\
\hline $\mathrm{j} 5$ & 9.00 & 8.40 & 4.50 & 3.20 & $\mathrm{j} 22$ & 7.20 & 10.50 & 4.50 & 4.50 & j39 & 3.20 & 3.70 & 1.90 & 2.20 \\
\hline j6 & 9.60 & 4.00 & 10.80 & 5.50 & $\mathrm{j} 23$ & 6.25 & 5.20 & 5.50 & 5.00 & $\mathrm{j} 40$ & 3.00 & 4.80 & 5.00 & 5.20 \\
\hline j7 & 7.60 & 6.00 & 3.00 & 6.40 & $\mathrm{j} 24$ & 11.20 & 13.60 & 15.40 & 12.00 & $\mathrm{j} 41$ & 10.20 & 3.60 & 4.20 & 10.80 \\
\hline j8 & 14.00 & 14.70 & 16.80 & 16.80 & $\mathrm{j} 25$ & 9.00 & 8.40 & 4.50 & 3.20 & $\mathrm{j} 42$ & 7.20 & 10.50 & 4.50 & 4.50 \\
\hline j9 & 4.80 & 3.00 & 6.30 & 3.60 & j26 & 9.60 & 4.00 & 10.80 & 5.50 & $\mathrm{j} 43$ & 6.25 & 5.20 & 5.50 & 5.00 \\
\hline $\mathrm{j} 10$ & 7.80 & 5.70 & 4.80 & 7.20 & $\mathrm{j} 27$ & 7.60 & 6.00 & 3.00 & 6.40 & $\mathrm{j} 44$ & 11.20 & 13.60 & 15.40 & 12.00 \\
\hline $\mathrm{j} 11$ & 5.20 & 2.40 & 1.00 & 3.00 & $\mathrm{j} 28$ & 14.00 & 14.70 & 16.80 & 16.80 & $\mathrm{j} 45$ & 9.00 & 8.40 & 4.50 & 3.20 \\
\hline $\mathrm{j} 12$ & 8.40 & 4.80 & 4.25 & 3.50 & j29 & 4.80 & 3.00 & 6.30 & 3.60 & $\mathrm{j} 46$ & 9.60 & 4.00 & 10.80 & 5.50 \\
\hline $\mathrm{j} 13$ & 8.75 & 5.70 & 4.00 & 6.00 & $\mathrm{j} 30$ & 7.80 & 5.70 & 4.80 & 7.20 & $\mathrm{j} 47$ & 7.60 & 6.00 & 3.00 & 6.40 \\
\hline $\mathrm{j} 14$ & 10.80 & 11.20 & 7.20 & 7.50 & $\mathrm{j} 31$ & 5.20 & 2.40 & 1.00 & 3.00 & $\mathrm{j} 48$ & 14.00 & 14.70 & 16.80 & 16.80 \\
\hline $\mathrm{j} 15$ & 5.70 & 4.44 & 5.70 & 4.17 & $\mathrm{j} 32$ & 8.40 & 4.80 & 4.25 & 3.50 & $\mathrm{j} 49$ & 4.80 & 3.00 & 6.30 & 3.60 \\
\hline $\mathrm{j} 16$ & 10.20 & 16.00 & 6.50 & 12.60 & $\mathrm{j} 33$ & 8.75 & 5.70 & 4.00 & 6.00 & $\mathrm{j} 50$ & 7.80 & 5.70 & 4.80 & 7.20 \\
\hline $\mathrm{j} 17$ & 4.55 & 17.50 & 5.50 & 5.25 & $\mathrm{j} 34$ & 10.80 & 11.20 & 7.20 & 7.50 & $u r_{u}$ & 0 & 0 & 0 & 0 \\
\hline
\end{tabular}

Table A-3 Changeover time $\left(c_{i j}\right)$ of order pairs

\begin{tabular}{|l|l|l|l|l|l|l|l|l|l|l|l|l|l|l|l|l|l|}
\hline & $\mathrm{j} 1$ & $\mathrm{j} 2$ & $\mathrm{j} 3$ & $\mathrm{j} 4$ & $\mathrm{j} 5$ & $\mathrm{j} 6$ & $\mathrm{j} 7$ & $\mathrm{j} 8$ & $\mathrm{j} 9$ & $\mathrm{j} 10$ & $\mathrm{j} 11$ & $\mathrm{j} 12$ & $\mathrm{j} 13$ & $\mathrm{j} 14$ & $\mathrm{j} 15$ & $\mathrm{j} 16$ & $\mathrm{j} 17$ \\
\hline $\mathrm{i} 1$ & 0.00 & 1.00 & 0.15 & 1.10 & 2.00 & 0.65 & 0.30 & 1.20 & 0.85 & 0.40 & 0.50 & 0.60 & 0.35 & 0.70 & 0.80 & 0.90 & 1.00 \\
\hline $\mathrm{i} 2$ & 1.80 & 0.00 & 1.10 & 1.30 & 1.40 & 0.90 & 0.20 & 1.20 & 0.40 & 0.30 & 0.25 & 0.50 & 0.70 & 0.75 & 1.00 & 0.25 & 1.25 \\
\hline i3 & 1.00 & 0.15 & 0.00 & 1.20 & 1.50 & 2.10 & 0.30 & 1.80 & 1.60 & 0.20 & 0.50 & 0.75 & 1.10 & 1.90 & 1.80 & 0.90 & 0.60 \\
\hline i4 & 1.20 & 0.02 & 0.10 & 0.00 & 0.05 & 1.60 & 1.20 & 2.00 & 1.20 & 0.50 & 1.20 & 0.80 & 1.00 & 0.70 & 0.45 & 1.70 & 1.30 \\
\hline i5 & 0.10 & 0.20 & 0.30 & 0.30 & 0.00 & 0.70 & 0.90 & 0.60 & 1.00 & 0.90 & 1.50 & 0.90 & 1.30 & 0.80 & 1.70 & 1.00 & 0.50 \\
\hline i6 & 1.40 & 0.80 & 0.30 & 0.70 & 2.00 & 0.00 & 0.90 & 1.20 & 1.20 & 1.60 & 0.20 & 0.40 & 1.20 & 0.55 & 0.20 & 1.00 & 0.35 \\
\hline
\end{tabular}




\begin{tabular}{|c|c|c|c|c|c|c|c|c|c|c|c|c|c|c|c|c|c|}
\hline i7 & 20 & 80 & 30 & .90 & 85 & 80 & 00 & 0.45 & 1.20 & 30 & .00 & 1.10 & 0.70 & .80 & 0.90 & .80 & 0.50 \\
\hline i8 & 30 & 1.40 & 50 & 1.40 & ) & 1.30 & .65 & 0.00 & 1.30 & & 70 & 1.05 & 0 & 0 & 0 & 0 & .90 \\
\hline i9 & 10 & 2. & 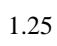 & 1.35 & 1.45 & 30 & 60 & 0.80 & 0.00 & 55 & 20 & 0.40 & 0.85 & 0.60 & 0.80 & 1.20 & 0.15 \\
\hline i10 & .50 & 1.20 & 60 & 78 & .50 & .40 & 90 & 0. & 0.70 & 0.00 & 1.90 & 1.00 & 1.15 & 31 & 0.95 & 0.50 & 0.40 \\
\hline i11 & 20 & 95 & 10 & 20 & 0 & 30 & 90 & 0 & 0 & 1.50 & 000 & ( & 0.15 & 2.00 & ) & 35 & 1.00 \\
\hline i12 & 1.30 & 0.7 & 0 & 0.60 & 0.40 & 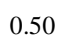 & 10 & 0. & 1.20 & 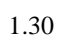 & . & $0 .($ & 1.50 & 1.40 & 1.30 & 00 & 0.80 \\
\hline i13 & 0.30 & 0 & 30 & 0.50 & 0.60 & 30 & 55 & 070 & 0.25 & 1.15 & 1.40 & 0.40 & 00 & 1.00 & 0.90 & 0.50 & 0.25 \\
\hline i14 & 0.50 & 0.70 & 30 & 0.9 & 5 & 0.80 & 50 & 1.00 & 0 & 1.20 & 0.20 & 0. & 0.60 & 0.00 & & & .00 \\
\hline i15 & 0.60 & 1.20 & 0 & 0.20 & 1.00 & 0.40 & 1.20 & 0. & 0.90 & 0.80 & 1.80 & 0 & 50 & 1.05 & 0.00 & 20 & .60 \\
\hline i16 & 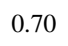 & 0.25 & 1.05 & .90 & 50 & 1.30 & 180 & 0.50 & 0 & 090 & 0.50 & 0 & 0.20 & 060 & ) & 0.00 & 0.80 \\
\hline i17 & 1.10 & 0.50 & 0.80 & 0.60 & 0.30 & 0. & 10 & 0 . & 0.70 & & 1.20 & & 0.45 & 0.80 & & 60 & .00 \\
\hline i18 & 0.40 & 1.80 & 60 & 0.50 & 0.45 & 1.50 & 20 & 1. & 35 & 0.60 & 0.70 & 0 & 90 & 0.65 & 5 & 00 & 0.30 \\
\hline i19 & 1.00 & 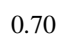 & 0.80 & 0.90 & 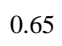 & 0.85 & 080 & 1.00 & 20 & 040 & 070 & c & 0.70 & 050 & 1.05 & 0.75 & .90 \\
\hline i 20 & 20 & 08 & ( & 0.15 & 0.60 & 0 & 55 & 0 & 1 & 0.40 & 1.20 & & 1.00 & 0.48 & 0 & 0.80 & 0.70 \\
\hline i21 & 1.60 & 1.00 & 0 & 1.10 & 2.00 & 0.65 & 30 & 1.20 & 0.85 & 0.40 & 0.5 & 0.60 & 35 & 0.70 & 0.80 & 90 & 1.00 \\
\hline i22 & ov & 60 & 10 & 1.30 & 40 & 0.90 & 20 & 1.2 & 0.40 & 0.50 & $0.2 J$ & 0. & 0.70 & 0.75 & 1.00 & 25 & 1.25 \\
\hline 23 & 1.00 & 0.15 & 160 & 1.20 & 1.50 & 2.10 & 030 & 180 & 1.60 & 020 & 050 & & 110 & 100 & & & 60 \\
\hline i24 & 1.20 & 1.62 & 0.10 & 1.60 & 0.05 & 1.60 & 1.20 & 2.00 & 1.20 & 50 & 1.20 & 0.80 & 00 & .70 & 0.4 & 70 & 1.30 \\
\hline i 25 & 10 & 20 & 30 & 0.30 & 1.60 & 0.10 & .90 & 0.0 & 1.00 & 0.90 & 1.5 & 0 . & 1.30 & 0.80 & 1.70 & 0 & 0.50 \\
\hline 6 & 1.40 & 0.80 & 0.30 & 0.70 & 2.00 & 1.60 & 000 & 100 & 1.20 & 160 & מ & & 120 & 0.55 & & & 35 \\
\hline i27 & 1.20 & 1 & 1.30 & 0.90 & 0.85 & 0 & 160 & 0 & 1 & 130 & 1.00 & 1 & 0.70 & Q8 & & & .50 \\
\hline i 28 & 1.30 & 1.40 & 50 & 1.40 & 1.20 & 1.30 & 65 & 1.60 & 1.30 & .80 & 1.70 & 05 & 1.50 & .30 & & 10 & 0.90 \\
\hline i 29 & 2.10 & 0 & & 1.35 & 1.45 & 0.80 & 60 & & 1.60 & 0.65 & & & 08 & .60 & & 1.20 & .15 \\
\hline 150 & 1.50 & (2) & 0.60 & 0.75 & 0.50 & 0.40 & م00 & 0.60 & & $1<0$ & n & & 115 & 20 & & & 40 \\
\hline i31 & 120 & 95 & 10 & 120 & 090 & 0.30 & 00 & 0 & 2 & 150 & 160 & 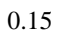 & 0.15 & 2.00 & 15 & 0.35 & 1.00 \\
\hline i32 & 1.30 & 0.70 & 0.80 & 0.60 & 0.40 & 0.50 & 10 & 0.20 & 1.20 & 1.30 & 0.60 & 1.00 & 1.5 & 1.40 & 1.3 & 1.00 & 0.80 \\
\hline i33 & م & 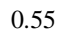 & & $-\sigma$ & 0 & 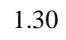 & & & & & & & & & & & 25 \\
\hline i34 & 0.50 & 70 & 0.80 & 0.90 & 1.45 & 0.80 & $0-5$ & 100 & 1.10 & 10 & & 040 & & 160 & & & .00 \\
\hline i35 & 0.60 & 1.20 & 10 & 0.20 & 1.00 & 0.40 & 20 & 0.30 & 0.90 & 80 & 1.8 & 0 & 15 & 1.05 & ) & 2 & 0.60 \\
\hline i36 & & & & 0.90 & 0.50 & & .00 & & & & & & & & & & 80 \\
\hline i37 & 1 & 0.5 & 8 & 060 & th & 0.9 & 111 & 0.50 & 070 & 075 & 1 & 100 & & 0 & & & 60 \\
\hline$i 38$ & 40 & 20 & 60 & 50 & 45 & 1.50 & 20 & 1.00 & 035 & 6 & 0.70 & 0 & 0.90 & 06 & 0.55 & 1.00 & 0.30 \\
\hline i39 & 1.00 & 0.70 & 0 & 0.90 & 0.65 & 0.8 & .80 & & & 0.4 & & & & 0.50 & & & .90 \\
\hline i40 & 2.00 & 0.80 & 0.60 & 0.15 & 0.60 & ( & (.JJ) & & & 0.40 & & & & 0.40 & & & 70 \\
\hline i41 & 1.60 & 1.00 & $1-$ & 110 & 00 & G & 2 & 1) & 005 & 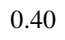 & 5 & 0 & 02 & 7( & 0 & 0.90 & 1.00 \\
\hline i42 & 181 & 1.60 & & 1.30 & 4 & 0.9 & 20 & 1.2 & 0.4 & 03 & & & & 0.7 & & & 1.25 \\
\hline $14 \mathrm{~J}$ & 1.00 & 0.15 & 1.00 & 1.20 & $1 .$. & 2.1 & 0.30 & & 1.0 & 0.2 & & & & & & & 0.60 \\
\hline$\therefore$ & 120 & 160 & . & 1 & 505 & 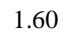 & 0 & 0 & 1 & $0 \leq 5$ & , & 00 & 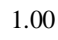 & 7 & 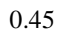 & 1.70 & 1.30 \\
\hline i45 & 01 & 0.20 & c & 0.30 & 1.60 & 0.7 & 0.90 & 0.60 & 1. & 0.90 & 1.50 & & 1.30 & 0.80 & & & 50 \\
\hline i46 & 1.40 & 0.80 & & 0.70 & 2.00 & & 0.90 & 1.20 & 1.20 & 1. & 0.2 & & 1.20 & 0.55 & 0.20 & 1.00 & 0.35 \\
\hline 147 & 1.20 & 1.80 & 1.30 & 0.90 & 0.85 & 0.00 & 00 & 0.45 & 1.20 & 1.00 & 1.00 & 1.10 & 0.70 & 0.00 & 0.90 & .80 & 0.50 \\
\hline i48 & 1.30 & 1.40 & 1.50 & 1.40 & 1.20 & 1.30 & 1.65 & & 1. & 0.80 & 1.70 & 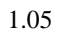 & 1.50 & 1 & & 1.10 & 90 \\
\hline i49 & & & & & & & 60 & & 1.60 & 0.65 & 0.20 & 0.40 & 0.85 & 0.60 & 0.80 & 1.20 & 0.15 \\
\hline
\end{tabular}




\begin{tabular}{|c|c|c|c|c|c|c|c|c|c|c|c|c|c|c|c|c|c|}
\hline i50 & 1.50 & 1.20 & 0.60 & 0.75 & 0.50 & 0.40 & 0.90 & 0.60 & 0.70 & 1.60 & 1.90 & 1.00 & 1.15 & 1.30 & 0.95 & 0.50 & 0.40 \\
\hline+ & $\mathrm{j} 18$ & j19 & $\mathrm{j} 20$ & $\mathrm{j} 21$ & $\mathrm{j} 22$ & $\mathrm{j} 23$ & j24 & $\mathrm{j} 25$ & j26 & $\mathrm{j} 27$ & $\mathrm{j} 28$ & j29 & $\mathrm{j} 30$ & j31 & $\mathrm{j} 32$ & $\mathrm{j} 33$ & j34 \\
\hline i1 & .65 & .20 & .40 & 1.60 & 1.00 & .15 & .10 & 2.00 & 0.65 & 0.30 & 1.20 & 0.85 & 0.40 & 0.50 & 0.60 & 0.35 & 0.70 \\
\hline i2 & 1.00 & 0.30 & 0.50 & 1.80 & 1.60 & 10 & .30 & 1.40 & 0.90 & 0.20 & 1.20 & 0.40 & 30 & 0.25 & 50 & 70 & .75 \\
\hline i3 & 0.20 & 0.40 & 0.70 & 1.00 & 0.15 & 1.60 & .20 & 1.50 & 2.10 & 0.30 & 1.80 & 1.60 & 0.20 & 0.50 & 0.75 & 1.10 & 1.90 \\
\hline i4 & 0.50 & 1.90 & 0.75 & 1.20 & 1.62 & 0.10 & 1.60 & 0.05 & 1.60 & 1.20 & 2.00 & 1.20 & 0.50 & 1.20 & 0.80 & 1.00 & 0.70 \\
\hline i5 & 0.70 & 1.30 & 0.80 & 0.10 & 0.20 & 0.30 & 0.30 & 1.60 & 0.70 & 0.90 & 0.60 & 1.00 & 0.90 & 1.50 & 0.90 & 1.30 & .80 \\
\hline i6 & 0.90 & 0.90 & 0.80 & 1.40 & 0.80 & 0.30 & 0.70 & 2.00 & 1.60 & 0.90 & 1.20 & 1.20 & 1.60 & 0.20 & 0.40 & 1.20 & 0.55 \\
\hline i7 & 1.00 & 0.80 & 0.25 & 1.20 & 1.80 & 1.30 & 0.90 & 0.85 & 0.80 & 1.60 & 0.45 & 1.20 & 1.30 & 1.00 & 1.10 & 0.70 & 0.80 \\
\hline i8 & 0.70 & 0.50 & 0.30 & 1.30 & 1.40 & 1.50 & 1.40 & 1.20 & 1.30 & 1.65 & 1.60 & 1.30 & 0.80 & 1.70 & 1.05 & 1.50 & 1.30 \\
\hline i9 & 1.20 & 1.00 & 0.80 & 2.10 & 2.00 & 1.25 & 1.35 & 1.45 & 0.80 & 1.60 & 0.80 & 1.60 & 0.65 & 0.20 & 0.40 & 0.85 & 0.60 \\
\hline i10 & 1.00 & 0.30 & 0.10 & 1.50 & 1.20 & 0.60 & 0.75 & 0.50 & 0.40 & 0.90 & 0.60 & 0.70 & 1.60 & 1.90 & 1.00 & 1.15 & 1.30 \\
\hline i11 & 0.90 & 0.20 & 0.80 & 1.20 & 0.95 & 1.10 & 1.20 & 0.90 & 0.30 & 0.90 & 0.80 & 2.00 & 1.50 & 1.60 & 0.15 & 0.15 & 2.00 \\
\hline i12 & 1.30 & 0.95 & 1.20 & 1.30 & 0.70 & 0.80 & 0.60 & 0.40 & 0.50 & 0.10 & 0.20 & 1.20 & 1.30 & 0.60 & 1.60 & 1.50 & 1.40 \\
\hline i13 & 0.80 & 0.35 & 0.70 & 0.30 & 0.55 & 1.30 & 0.50 & 0.60 & 1.30 & 55 & 0.70 & 0.25 & 15 & .40 & 0.40 & 1.60 & .00 \\
\hline i14 & 0.55 & 0.50 & 0.65 & 0.50 & 0.70 & 0.80 & 90 & 1.45 & 0.80 & 50 & 1.00 & 1.10 & .20 & 0.20 & 0.40 & 0.60 & .60 \\
\hline i15 & 0.30 & 0.90 & 0.60 & 0.60 & 1.20 & 1.10 & 0.20 & 1.00 & 0.40 & 1.20 & 0.30 & 0.90 & 0.80 & 1.80 & 0.30 & 1.50 & 1.05 \\
\hline i16 & 0.90 & 0.15 & 1.00 & 0.70 & 0.25 & 1.05 & 0.90 & 0.50 & 1.30 & 1.80 & 0.50 & 0.80 & 0.90 & 0.50 & 0.85 & 0.20 & 0.60 \\
\hline i17 & 0. & 0.40 & 0 & 1.10 & 0.50 & 0.80 & 60 & 0 & 0 & 10 & 0.50 & 70 & 75 & .20 & 1.00 & .45 & .80 \\
\hline i18 & 0.00 & 0.60 & 0.45 & 0.40 & 1.80 & 1.60 & 0.50 & 0.45 & 1.50 & 1.20 & 1.00 & 0.35 & 0.60 & 0.70 & 0.80 & 0.90 & 0.65 \\
\hline i19 & 0.45 & 0.00 & 0.01 & 1.00 & 0.70 & 0.80 & 0.90 & 0.65 & 0.85 & 0.80 & 1.00 & 1.20 & 0.40 & 0.70 & 0.90 & 0.70 & 0.50 \\
\hline i20 & 0.60 & 0.50 & 0.00 & 2.00 & 0.80 & 0.60 & 15 & 0.60 & 0.90 & 0.55 & 0.45 & 1.30 & 0.40 & 1.20 & 1.10 & 1.00 & 0.40 \\
\hline i 21 & 0.65 & 1.20 & 1.40 & 0.00 & 1.00 & 0.15 & 1.10 & 2.0 & 0.65 & 0.31 & 1.20 & 0.85 & 0.40 & 0.50 & 0.60 & 0.35 & 0.70 \\
\hline i22 & 1.00 & 0.30 & 0.50 & 1.80 & 0.00 & 1.10 & 1.30 & 1.40 & 0.90 & 0.20 & 1.20 & 0.40 & 0.30 & 0.25 & 0.50 & 0.70 & 0.75 \\
\hline i23 & 0.20 & 0.40 & 0.70 & 1.00 & 0.15 & 0.00 & 1.20 & 1.50 & 2.10 & 0.30 & 1.80 & 1.60 & 0.20 & 0.50 & 0.75 & 1.10 & 1.90 \\
\hline i24 & 0. & 1.90 & 0.75 & 12 & 0.02 & (10 & 0.00 & م & 1.60 & 10 & 2.00 & 1.20 & 0.50 & 1.20 & 0 & 00 & 0.70 \\
\hline i 25 & 0.70 & 1.30 & 0.80 & 0.10 & 0.20 & 0.30 & 0.30 & 0.00 & 0.70 & 0.90 & 0.60 & 1.00 & 0.90 & 1.50 & 0.90 & 1.30 & 0.80 \\
\hline i26 & 0.90 & 0.90 & 0.80 & 1.40 & 0.80 & 0.30 & 0.70 & 2.00 & 0.00 & 0.90 & 1.20 & 1.20 & 1.60 & 0.20 & 0.40 & 1.20 & 0.55 \\
\hline i27 & 1.00 & 0.80 & 0.25 & 1.20 & 1.80 & 1.30 & 90 & 0.85 & 0.80 & 0.0 & 0.4 & 20 & 1.30 & 1.0 & 1.10 & 0.70 & 80 \\
\hline i 28 & 0.70 & 0.50 & 0 & 1 & 1.40 & 1 & 40 & 0 & 0 & 1.65 & 0.00 & 0 & 80 & 1.70 & 5 & 1.50 & .30 \\
\hline i29 & 1.20 & 1.00 & 0.80 & 2.10 & 2.00 & 1.25 & 1.35 & 1.45 & 0.80 & 1.60 & 0.80 & 0.00 & 0.65 & 0.20 & 0.40 & 0.85 & 0.60 \\
\hline i30 & 1.00 & 0.30 & 0.10 & 1.50 & 1.20 & 0.60 & 0.75 & 0.50 & 0.40 & 0.90 & 0.60 & 0.70 & 0.00 & 1.90 & 1.00 & 1.15 & 1.30 \\
\hline i31 & 0.9 & 0.20 & 0 & 1.2 & 0. & 1 & 20 & 0.90 & 0 & 0.90 & 0 & 0 & 0 & 0.00 & 0.15 & 5 & .00 \\
\hline i32 & 1.30 & 0.95 & 1.20 & 1.30 & 0.70 & 0.80 & 0.60 & 0.40 & 0.50 & 0.10 & 0.20 & 1.20 & 1.30 & 0.60 & 0.00 & 1.50 & 1.40 \\
\hline i33 & 0.80 & 0.35 & 0.70 & 0.30 & 0.55 & 1.30 & 0.50 & 0.60 & 1.30 & 1.55 & 0.70 & 0.25 & 1.15 & 1.40 & 0.40 & 0.00 & 1.00 \\
\hline i34 & 0.55 & 0.50 & 0.65 & 0.50 & 0.70 & 0.80 & 0.90 & 1.45 & 0.80 & 0.5 & 1. & 1.10 & 1.20 & 0.20 & 0. & 0.60 & 0.00 \\
\hline i35 & 0.30 & 0.90 & 0.60 & 0.60 & 1.20 & 1.10 & 0.20 & 1.00 & 0.40 & 1.20 & 0.30 & 0.90 & 0.80 & 1.80 & 0.30 & 1.50 & 1.05 \\
\hline i36 & 0.90 & 0.15 & 1.00 & 0.70 & 0.25 & 1.05 & 0.90 & 0.50 & 1.30 & 1.80 & 0.50 & 0.80 & 0.90 & 0.50 & 0.85 & 0.20 & 0.60 \\
\hline i37 & 0.15 & 0.40 & 0.30 & 1.10 & 0.50 & 0.80 & 0.60 & 0.30 & 0.90 & 1.10 & 0.50 & 0.70 & 0.75 & 1.20 & 1.00 & 0.45 & 0.80 \\
\hline i38 & 1.60 & 0.60 & 0.45 & 0.40 & 1.80 & 1.60 & .50 & 0. & 1.50 & 1.20 & 1.0 & 0.35 & 0.60 & 0.70 & 0.80 & 0.90 & 0.65 \\
\hline i39 & 0.45 & 1.60 & 0.01 & 1.00 & 0.70 & 0 & 90 & 0.65 & 5 & 30 & 1.0 & 1.20 & 40 & 0.70 & 0.90 & 0.70 & .50 \\
\hline i 40 & 0.60 & 0.50 & 1.60 & 2.00 & 0.80 & 0.60 & 0.15 & 0.60 & 0.90 & 0.55 & 0.45 & 1.30 & 0.40 & 1.20 & 1.10 & 1.00 & 0.40 \\
\hline i41 & 0.65 & 1.20 & 1.40 & 1.60 & 1.00 & 0.15 & 1.10 & 2.00 & 0.65 & 0.30 & 1.20 & 0.85 & 0.40 & 0.50 & 0.60 & 0.35 & 0.70 \\
\hline
\end{tabular}




\begin{tabular}{|c|c|c|c|c|c|c|c|c|c|c|c|c|c|c|c|c|c|}
\hline i42 & 1.00 & 0.30 & 0.50 & 1.80 & 1.60 & 1.10 & 1.30 & 1.40 & 0.90 & 0.20 & 1.20 & 0.40 & 0.30 & 0.25 & 0.50 & 0.70 & 0.75 \\
\hline i43 & 0.20 & 0.40 & 0.70 & 1.00 & 0.15 & 1.60 & 1.20 & 1.50 & 2.10 & 0.30 & 1.80 & 1.60 & 0.20 & 0.50 & 0.75 & 1.10 & 1.90 \\
\hline i44 & 0.50 & 1.90 & 0.75 & 1.20 & 1.62 & 0.10 & 1.60 & 0.05 & 1.60 & 1.20 & 2.00 & 1.20 & 0.50 & 1.20 & 0.80 & 1.00 & 0.70 \\
\hline i45 & 0.70 & 1.30 & 0.80 & 0.10 & 0.20 & 0.30 & 0.30 & 1.60 & 0.70 & 0.90 & 0.60 & 1.00 & 0.90 & 1.50 & 0.90 & 1.30 & 0.80 \\
\hline i46 & 0.90 & 0.90 & 0.80 & 1.40 & 0.80 & 0.30 & 0.70 & 2.00 & 1.60 & 0.90 & 1.20 & 1.20 & 1.60 & 0.20 & 0.40 & 1.20 & 0.55 \\
\hline i47 & 1.00 & 0.80 & 0.25 & 1.20 & 1.80 & 1.30 & 0.90 & 0.85 & 0.80 & 1.60 & 0.45 & 1.20 & 1.30 & 1.00 & 1.10 & 0.70 & 0.80 \\
\hline i48 & 0.70 & 0.50 & 0.30 & 1.30 & 1.40 & 1.50 & 1.40 & 1.20 & 1.30 & 1.65 & 1.60 & 1.30 & 0.80 & 1.70 & 1.05 & 1.50 & 1.30 \\
\hline i49 & 1.20 & 1.00 & 0.80 & 2.10 & 2.00 & 1.25 & 1.35 & 1.45 & 0.80 & 1.60 & 0.80 & 1.60 & 0.65 & 0.20 & 0.40 & 0.85 & 0.60 \\
\hline i50 & 1.00 & 0.30 & 0.10 & 1.50 & 1.20 & 0.60 & 0.75 & 0.50 & 0.40 & 0.90 & 0.60 & 0.70 & 1.60 & 1.90 & 1.00 & 1.15 & 1.30 \\
\hline+ & j35 & $\mathrm{j} 36$ & $\mathrm{j} 37$ & $\mathrm{j} 38$ & j39 & $\mathrm{j} 40$ & $\mathrm{j} 41$ & $\mathrm{j} 42$ & $\mathrm{j} 43$ & j44 & $\mathrm{j} 45$ & j46 & $\mathrm{j} 47$ & j48 & j49 & j50 & \\
\hline i1 & 0.80 & 0.90 & 1.00 & 0.65 & 1.20 & 1.40 & 1.20 & 0.95 & 1.10 & 1.20 & 0.90 & 0.30 & 0.90 & 0.80 & 2.00 & 1.50 & \\
\hline i2 & 1.00 & 0.25 & 1.25 & 1.00 & 0.30 & 0.50 & 1.30 & 0.70 & 0.80 & 0.60 & 0.40 & 0.50 & 0.10 & 0.20 & 1.20 & 1.30 & \\
\hline i3 & 1.80 & 0.90 & 0.60 & 0.20 & 0.40 & 0.70 & 0.30 & 0.55 & 1.30 & 0.50 & 0.60 & 1.30 & 1.55 & 0.70 & 0.25 & 1.15 & \\
\hline i4 & 0.45 & 1.70 & 1.30 & 0.50 & 1.90 & 0.75 & 0.50 & 0.70 & 0.80 & 0.90 & 1.45 & 0.80 & 0.50 & 1.00 & 1.10 & 1.20 & \\
\hline i5 & 1.70 & 1.00 & 0.50 & 0.70 & 1.30 & 0.80 & 0.60 & 1.20 & 1.10 & 0.20 & 1.00 & 0.40 & 1.20 & 0.30 & 0.90 & 0.80 & \\
\hline i6 & 0.20 & 1.00 & 0.35 & 0.90 & 0.90 & 0.80 & 0.70 & 0.25 & 1.05 & 0.90 & 0.50 & 1.30 & 1.80 & 0.50 & 0.80 & 0.90 & \\
\hline i7 & 0.90 & 0.80 & 0.50 & 1.00 & 0.80 & 0.25 & 1.10 & 0.50 & 0.80 & 0.60 & 0.30 & 0.90 & 1.10 & 0.50 & 0.70 & 0.75 & \\
\hline i8 & 1.10 & 1.10 & 0.90 & 0.70 & 0.50 & 0.30 & 0.40 & 1.80 & 1.60 & 0.50 & 0.45 & 1.50 & 1.20 & 1.00 & 0.35 & 0.60 & \\
\hline i9 & 0.80 & 1.20 & 0.15 & 1.20 & 1.00 & 0.80 & 1.00 & 0.70 & 0.80 & 0.90 & 0.65 & 0.85 & 0.80 & 1.00 & 1.20 & 0.40 & \\
\hline i10 & 0.95 & 0.50 & 0.40 & 1.00 & 0.30 & 0.10 & 2.00 & 0.80 & 0.60 & 0.15 & 0.60 & 0.90 & 0.55 & 0.45 & 1.30 & 0.40 & \\
\hline i11 & 1.50 & 0.35 & 1.00 & 0.90 & 0.20 & 0.80 & 1.60 & 1.00 & 0.15 & 1.10 & 2.00 & 0.65 & 0.30 & 1.20 & 0.85 & 0.40 & \\
\hline i12 & 1.30 & 1.00 & 0.80 & 1.30 & 0.95 & 1.20 & 1.80 & 1.60 & 1.10 & 1.30 & 1.40 & 0.90 & 0.20 & 1.20 & 0.40 & 0.30 & \\
\hline i13 & 0.90 & 0.50 & 0.25 & 0.80 & 0.35 & 0.70 & 1.00 & 0.15 & 1.60 & 1.20 & 1.50 & 2.10 & 0.30 & 1.80 & 1.60 & 0.20 & \\
\hline i14 & 0.75 & 0.80 & 1.00 & 0.55 & 0.50 & 0.65 & 1.20 & 1.62 & 0.10 & 1.60 & 0.05 & 1.60 & 1.20 & 2.00 & 1.20 & 0.50 & \\
\hline i15 & 1.60 & 1.20 & 0.60 & 0.30 & 0.90 & 0.60 & 0.10 & 0.20 & 0.30 & 0.30 & 1.60 & 0.70 & 0.90 & 0.60 & 1.00 & 0.90 & \\
\hline i16 & 0.70 & 1.60 & 0.80 & 0.90 & 0.15 & 1.00 & 1.40 & 0.80 & 0.30 & 0.70 & 2.00 & 1.60 & 0.90 & 1.20 & 1.20 & 1.60 & \\
\hline i17 & 0.20 & 0.60 & 1.60 & 0.15 & 0.40 & 0.30 & 1.20 & 1.80 & 1.30 & 0.90 & 0.85 & 0.80 & 1.60 & 0.45 & 1.20 & 1.30 & \\
\hline i18 & 0.55 & 1.00 & 0.30 & 1.60 & 0.60 & 0.45 & 1.30 & 1.40 & 1.50 & 1.40 & 1.20 & 1.30 & 1.65 & 1.60 & 1.30 & 0.80 & \\
\hline i19 & 1.05 & 0.75 & 0.90 & 0.45 & 1.60 & 0. & 2.10 & 2.00 & 1. & 5 & 1.45 & 0. & 1.60 & 0.80 & 1.60 & 0.6 & \\
\hline i20 & 0.90 & 0.80 & 0.70 & 0.60 & 0.50 & 1.60 & 1.50 & 1.20 & 0.60 & 0.75 & 0.50 & 0.40 & 0.90 & 0.60 & 0.70 & 1.60 & \\
\hline i21 & 0.80 & 0.90 & 1.00 & 0.65 & 1.20 & 1.40 & 1.20 & 0.95 & 1.10 & 1.20 & 0.90 & 0.30 & 0.90 & 0.80 & 2.00 & 1.50 & \\
\hline i22 & 1.00 & 0.25 & 1.25 & 1.00 & 0.30 & 0.50 & 1.30 & 0.70 & 0.80 & 0.60 & 0.40 & 0.50 & 0.10 & 0.20 & 1.20 & 1.30 & \\
\hline i23 & 1.80 & 0.90 & 0.60 & 0.20 & 0.40 & 0.70 & 0.30 & 0.55 & 1.30 & 0.50 & 0.60 & 1.30 & 1.55 & 0.70 & 0.25 & 1.15 & \\
\hline i24 & 0.45 & 1.70 & 1.30 & 0.50 & 1.90 & 0.75 & 0.50 & 0.70 & 0.80 & 0.90 & 1.45 & 0.80 & 0.50 & 1.00 & 1.10 & 1.20 & \\
\hline i25 & 1.70 & 1.00 & 0.50 & 0.70 & 1.30 & 0.80 & 0.60 & 1.20 & 1.10 & 0.20 & 1.00 & 0.40 & 1.20 & 0.30 & 0.90 & 0.80 & \\
\hline i26 & 0.20 & 1.00 & 0.35 & \begin{tabular}{|l|l} 
& 0.90
\end{tabular} & 0.90 & 0.80 & 0.70 & 0.25 & 1.05 & 0.90 & 0.50 & 1.30 & 1.80 & 0.50 & 0.80 & 0.90 & \\
\hline i27 & 0.90 & 0.80 & 0.50 & 1.00 & 0.80 & 0.25 & 1.10 & 0.50 & 0.80 & 0.60 & 0.30 & 0.90 & 1.10 & 0.50 & 0.70 & 0.75 & \\
\hline i28 & 1.10 & 1.10 & 0.90 & 0.70 & 0.50 & 0.30 & 0.40 & 1.80 & 1.60 & 0.50 & 0.45 & 1.50 & 1.20 & 1.00 & 0.35 & 0.60 & \\
\hline i29 & 0.80 & 1.20 & 0.15 & 1.20 & 1.00 & 0.80 & 1.00 & 0.70 & 0.80 & 0.90 & 0.65 & 0.85 & 0.80 & 1.00 & 1.20 & 0.40 & \\
\hline $\mathrm{i} 30$ & 0.95 & 0.50 & 0.40 & 1.00 & 0.30 & 0.10 & 2.00 & 0.80 & 0.60 & 0.15 & 0.60 & 0.90 & 0.55 & 0.45 & 1.30 & 0.40 & \\
\hline i31 & 1.50 & 0.35 & 1.00 & \begin{tabular}{|l|l} 
& 0.90
\end{tabular} & 0.20 & 0.80 & 1.60 & 1.00 & 0.15 & 1.10 & 2.00 & 0.65 & 0.30 & 1.20 & 0.85 & 0.40 & \\
\hline $\mathrm{i} 32$ & 1.30 & 1.00 & 0.80 & 1.30 & 0.95 & 1.20 & 1.80 & 1.60 & 1.10 & 1.30 & 1.40 & 0.90 & 0.20 & 1.20 & 0.40 & 0.30 & \\
\hline i33 & 0.90 & 0.50 & 0.25 & 0.80 & 0.35 & 0.70 & 1.00 & 0.15 & 1.60 & 1.20 & 1.50 & 2.10 & 0.30 & 1.80 & 1.60 & 0.20 & \\
\hline
\end{tabular}




\begin{tabular}{|l|l|l|l|l|l|l|l|l|l|l|l|l|l|l|l|l|l|}
\hline i34 & 0.75 & 0.80 & 1.00 & 0.55 & 0.50 & 0.65 & 1.20 & 1.62 & 0.10 & 1.60 & 0.05 & 1.60 & 1.20 & 2.00 & 1.20 & 0.50 & \\
\hline i35 & 0.00 & 1.20 & 0.60 & 0.30 & 0.90 & 0.60 & 0.10 & 0.20 & 0.30 & 0.30 & 1.60 & 0.70 & 0.90 & 0.60 & 1.00 & 0.90 & \\
\hline i36 & 0.70 & 0.00 & 0.80 & 0.90 & 0.15 & 1.00 & 1.40 & 0.80 & 0.30 & 0.70 & 2.00 & 1.60 & 0.90 & 1.20 & 1.20 & 1.60 & \\
\hline i37 & 0.20 & 0.60 & 0.00 & 0.15 & 0.40 & 0.30 & 1.20 & 1.80 & 1.30 & 0.90 & 0.85 & 0.80 & 1.60 & 0.45 & 1.20 & 1.30 & \\
\hline i38 & 0.55 & 1.00 & 0.30 & 0.00 & 0.60 & 0.45 & 1.30 & 1.40 & 1.50 & 1.40 & 1.20 & 1.30 & 1.65 & 1.60 & 1.30 & 0.80 & \\
\hline i39 & 1.05 & 0.75 & 0.90 & 0.45 & 0.00 & 0.01 & 2.10 & 2.00 & 1.25 & 1.35 & 1.45 & 0.80 & 1.60 & 0.80 & 1.60 & 0.65 & \\
\hline i40 & 0.90 & 0.80 & 0.70 & 0.60 & 0.50 & 0.00 & 1.50 & 1.20 & 0.60 & 0.75 & 0.50 & 0.40 & 0.90 & 0.60 & 0.70 & 1.60 & \\
\hline i41 & 0.80 & 0.90 & 1.00 & 0.65 & 1.20 & 1.40 & 0.00 & 1.00 & 0.15 & 1.10 & 2.00 & 0.65 & 0.30 & 1.20 & 0.85 & 0.40 & \\
\hline i42 & 1.00 & 0.25 & 1.25 & 1.00 & 0.30 & 0.50 & 1.80 & 0.00 & 1.10 & 1.30 & 1.40 & 0.90 & 0.20 & 1.20 & 0.40 & 0.30 & \\
\hline i43 & 1.80 & 0.90 & 0.60 & 0.20 & 0.40 & 0.70 & 1.00 & 0.15 & 0.00 & 1.20 & 1.50 & 2.10 & 0.30 & 1.80 & 1.60 & 0.20 & \\
\hline i44 & 0.45 & 1.70 & 1.30 & 0.50 & 1.90 & 0.75 & 1.20 & 0.02 & 0.10 & 0.00 & 0.05 & 1.60 & 1.20 & 2.00 & 1.20 & 0.50 & \\
\hline i45 & 1.70 & 1.00 & 0.50 & 0.70 & 1.30 & 0.80 & 0.10 & 0.20 & 0.30 & 0.30 & 0.00 & 0.70 & 0.90 & 0.60 & 1.00 & 0.90 & \\
\hline i46 & 0.20 & 1.00 & 0.35 & 0.90 & 0.90 & 0.80 & 1.40 & 0.80 & 0.30 & 0.70 & 2.00 & 0.00 & 0.90 & 1.20 & 1.20 & 1.60 & \\
\hline i47 & 0.90 & 0.80 & 0.50 & 1.00 & 0.80 & 0.25 & 1.20 & 1.80 & 1.30 & 0.90 & 0.85 & 0.80 & 0.00 & 0.45 & 1.20 & 1.30 & \\
\hline i48 & 1.10 & 1.10 & 0.90 & 0.70 & 0.50 & 0.30 & 1.30 & 1.40 & 1.50 & 1.40 & 1.20 & 1.30 & 1.65 & 0.00 & 1.30 & 0.80 & \\
\hline i49 & 0.80 & 1.20 & 0.15 & 1.20 & 1.00 & 0.80 & 2.10 & 2.00 & 1.25 & 1.35 & 1.45 & 0.80 & 1.60 & 0.80 & 0.00 & 0.65 & \\
\hline i50 & 0.95 & 0.50 & 0.40 & 1.00 & 0.30 & 0.10 & 1.50 & 1.20 & 0.60 & 0.75 & 0.50 & 0.40 & 0.90 & 0.60 & 0.70 & 0.00 & \\
\hline
\end{tabular}

\section{B. Data for Example 3}

Table B-1 order due date $\left(d_{j}\right)$ and order release time $\left(o r_{j}\right)$

\begin{tabular}{|c|c|c|c|c|c|c|c|c|c|c|c|c|c|c|c|c|c|}
\hline Order & $\mathrm{j} 1$ & $\mathrm{j} 2$ & $\mathrm{j} 3$ & $\mathrm{j} 4$ & $\mathrm{j} 5$ & $\mathrm{j} 6$ & $\mathrm{j} 7$ & $\mathrm{j} 8$ & $\mathrm{j} 9$ & $\mathrm{j} 10$ & $\mathrm{j} 11$ & $\mathrm{j} 12$ & $\mathrm{j} 13$ & $\mathrm{j} 14$ & $\mathrm{j} 15$ & $\mathrm{j} 16$ & $\mathrm{j} 17$ \\
\hline $\boldsymbol{d}_{\boldsymbol{j}}$ & 20 & 44 & 50 & 40 & 56 & 60 & 34 & 46 & 60 & 60 & 40 & 64 & 70 & 60 & 76 & 80 & 74 \\
\hline $\boldsymbol{o r}_{\boldsymbol{j}}$ & 0 & 5 & 0 & 6 & 0 & 2 & 3 & 0 & 2 & 6 & 0 & 1.5 & 0 & 0 & 5.5 & 0 & 3 \\
\hline \hline Order & $\mathrm{j} 18$ & $\mathrm{j} 19$ & $\mathrm{j} 20$ & $\mathrm{j} 21$ & $\mathrm{j} 22$ & $\mathrm{j} 23$ & $\mathrm{j} 24$ & $\mathrm{j} 25$ & $\mathrm{j} 26$ & $\mathrm{j} 27$ & $\mathrm{j} 28$ & $\mathrm{j} 29$ & $\mathrm{j} 30$ & $\mathrm{j} 31$ & $\mathrm{j} 32$ & $\mathrm{j} 33$ & $\mathrm{j} 34$ \\
\hline $\boldsymbol{d}_{\boldsymbol{j}}$ & 66 & 80 & 80 & 20 & 44 & 50 & 40 & 56 & 60 & 34 & 46 & 60 & 60 & 40 & 64 & 70 & 60 \\
\hline $\boldsymbol{o r}_{\boldsymbol{j}}$ & 0 & 2 & 0 & 4 & 5 & 0 & 6 & 0 & 2 & 3 & 0 & 2 & 6 & 0 & 1.5 & 0 & 0 \\
\hline \hline Order & $\mathrm{j} 35$ & $\mathrm{j} 36$ & $\mathrm{j} 37$ & $\mathrm{j} 38$ & $\mathrm{j} 39$ & $\mathrm{j} 40$ & $\mathrm{j} 41$ & $\mathrm{j} 42$ & $\mathrm{j} 43$ & $\mathrm{j} 44$ & $\mathrm{j} 45$ & $\mathrm{j} 46$ & $\mathrm{j} 47$ & $\mathrm{j} 48$ & $\mathrm{j} 49$ & $\mathrm{j} 50$ & \\
\hline $\boldsymbol{d}_{\boldsymbol{j}}$ & 76 & 80 & 74 & 66 & 80 & 80 & 20 & 44 & 50 & 40 & 56 & 60 & 34 & 46 & 60 & 60 & \\
\hline $\boldsymbol{o r}_{\boldsymbol{j}}$ & 5.5 & 0 & 5 & 0 & 6 & 0 & 2 & 3 & 0 & 2 & 6 & 0 & 1.5 & 0 & 0 & 5.5 & \\
\hline
\end{tabular}

Table B-2 Process time $\left(p_{i u}\right)$ and unit release time $\left(u r_{u}\right)$

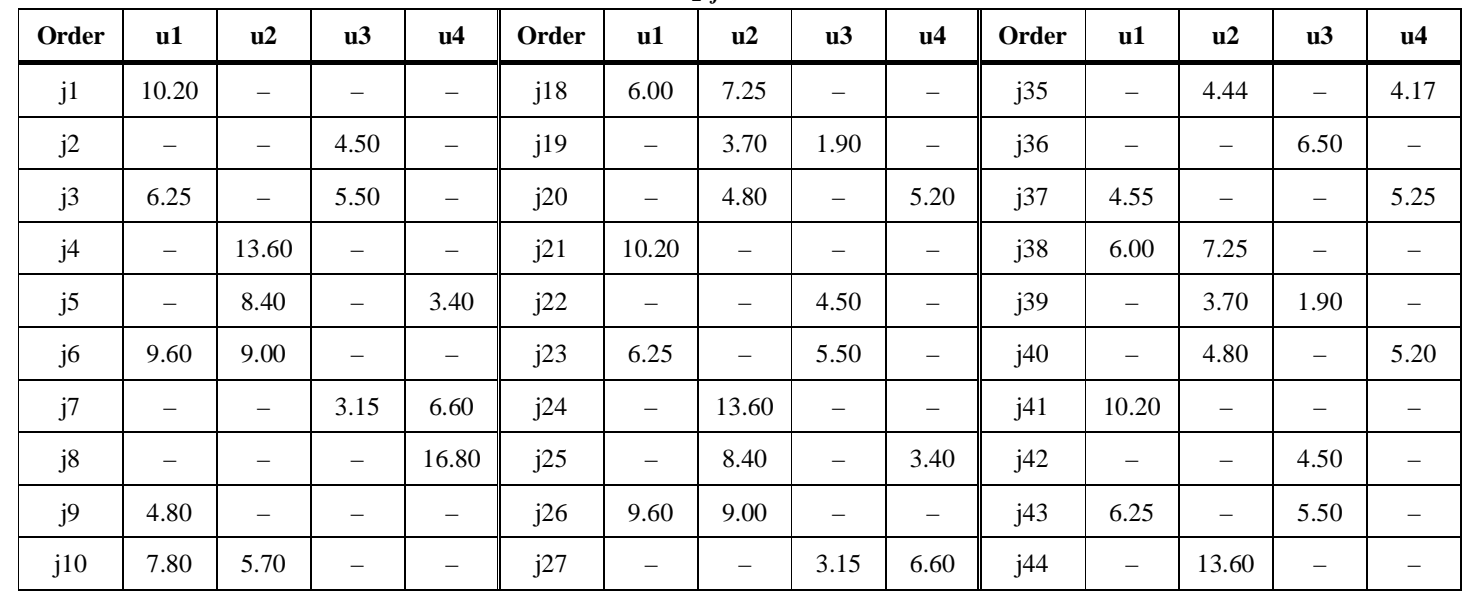




\begin{tabular}{|c|c|c|c|c||c|c|c|c|c||c|c|c|c|c|}
\hline $\mathrm{j} 11$ & - & - & 1.00 & - & $\mathrm{j} 28$ & - & - & - & 16.80 & $\mathrm{j} 45$ & - & 8.40 & - & 3.40 \\
\hline $\mathrm{j} 12$ & - & - & 3.40 & 2.10 & $\mathrm{j} 29$ & 4.80 & - & - & - & $\mathrm{j} 46$ & 9.60 & 9.00 & - & - \\
\hline $\mathrm{j} 13$ & 3.50 & - & 2.00 & - & $\mathrm{j} 30$ & 7.80 & 5.70 & - & - & $\mathrm{j} 47$ & - & - & 3.15 & 6.60 \\
\hline $\mathrm{j} 14$ & - & 11.20 & - & - & $\mathrm{j} 31$ & - & - & 1.00 & - & $\mathrm{j} 48$ & - & - & - & 16.80 \\
\hline $\mathrm{j} 15$ & - & 4.44 & - & 4.17 & $\mathrm{j} 32$ & - & - & 3.40 & 2.10 & $\mathrm{j} 49$ & 4.80 & - & - & - \\
\hline $\mathrm{j} 16$ & - & - & 6.50 & - & $\mathrm{j} 33$ & 3.50 & - & 2.00 & - & $\mathrm{j} 50$ & 7.80 & 5.70 & - & $-;$ \\
\hline $\mathrm{j} 17$ & 4.55 & - & - & 5.25 & $\mathrm{j} 34$ & - & 11.20 & - & - & $u r_{u}$ & 0 & 3 & 2 & 3 \\
\hline
\end{tabular}

Table B-3 Changeover time $\left(c_{i j}\right)$ of order pairs

\begin{tabular}{|c|c|c|c|c|c|c|c|c|c|c|c|c|c|c|c|c|c|}
\hline & $\mathrm{j} 1$ & $\mathrm{j} 2$ & j3 & $\mathrm{j} 4$ & j5 & j6 & j7 & j8 & j9 & $\mathrm{j} 10$ & $\mathrm{j} 11$ & $\mathrm{j} 12$ & $\mathrm{j} 13$ & $\mathrm{j} 14$ & $\mathrm{j} 15$ & j16 & $\mathrm{j} 17$ \\
\hline i1 & - & - & - & - & - & 0.65 & - & - & 0.85 & 0.40 & - & - & 0.35 & - & - & - & - \\
\hline i2 & - & - & 1.10 & - & - & - & - & - & - & - & 0.25 & - & 0.70 & - & - & 0.25 & - \\
\hline i3 & 1.00 & 0.15 & - & - & - & - & 0.30 & - & 1.60 & 0.20 & 0.50 & 0.75 & - & - & - & 0.90 & 0.60 \\
\hline i4 & - & - & - & - & 0.05 & - & - & - & - & 0.50 & - & - & - & 0.70 & 0.45 & - & - \\
\hline i5 & - & - & - & 0.30 & - & 0.70 & 0.90 & 0.60 & - & - & - & 0.90 & - & 0.80 & - & - & - \\
\hline i6 & 1.40 & - & 0.30 & 0.70 & - & - & - & - & 1.20 & - & - & - & 1.20 & 0.55 & 0.20 & - & 0.35 \\
\hline i7 & - & 1.80 & - & - & 0.85 & - & - & 0.45 & - & - & 1.00 & 1.10 & - & - & - & 0.80 & 0.50 \\
\hline i8 & - & - & - & - & - & - & 1.65 & - & - & - & - & 1.05 & - & - & 1.10 & - & - \\
\hline i9 & 2.10 & - & 1.25 & - & - & 0.80 & - & - & - & 0.65 & - & - & 0.85 & - & - & - & 0.15 \\
\hline $\mathrm{i} 10$ & 1.50 & - & 0.60 & 0.75 & 0.50 & - & - & - & 0.70 & - & - & - & 1.15 & 1.30 & 0.95 & - & 0.40 \\
\hline i11 & - & 0.95 & - & - & - & - & - & - & - & - & - & 0.15 & 0.15 & - & - & 0.35 & - \\
\hline i12 & - & - & 0.80 & - & 0.40 & - & 0.10 & 0.20 & - & - & 0.60 & - & - & - & 1.30 & 1.00 & 0.80 \\
\hline i13 & 0.30 & 0.55 & 1.30 & - & - & 1.30 & 1.55 & - & 0.25 & 1.15 & 1.40 & 0.40 & - & - & - & 0.50 & 0.25 \\
\hline i14 & - & - & - & 1.45 & 0.80 & 0.50 & - & - & - & 0.35 & - & - & - & - & 0.75 & - & - \\
\hline i15 & - & - & - & 0.20 & - & 0.40 & 1.20 & 0.30 & - & 0.80 & - & 0.30 & - & 1.05 & - & - & 0.60 \\
\hline i16 & - & 0.25 & 1.05 & - & - & - & - & - & - & - & - & 0.85 & 0.20 & - & - & - & - \\
\hline i17 & - & - & 0.80 & - & 0.30 & 0.90 & 1.10 & 0.50 & - & 0.75 & - & - & 0.45 & - & 0.20 & - & - \\
\hline i18 & 0.40 & - & - & 0.50 & 0.45 & - & - & - & 0.35 & 0.60 & - & - & - & 0.65 & 0.55 & - & 0.30 \\
\hline i19 & - & 0.70 & - & - & 0.65 & 0.85 & 0.80 & - & - & - & 0.70 & 0.90 & - & 0.50 & 1.05 & 0.75 & - \\
\hline $\mathrm{i} 20$ & - & - & - & 0.15 & - & - & 0.55 & 0.45 & - & 0.40 & - & - & - & 0.40 & - & - & - \\
\hline $\mathrm{i} 21$ & - & - & - & - & - & 0.65 & - & - & 0.85 & 0.40 & - & - & 0.35 & - & - & - & - \\
\hline i22 & - & - & 1.10 & - & - & - & - & - & - & - & 0.25 & - & 0.70 & - & - & 0.25 & - \\
\hline i23 & 1.00 & 0.15 & - & - & - & - & 0.30 & - & 1.60 & 0.20 & 0.50 & 0.75 & - & - & - & 0.90 & 0.60 \\
\hline $\mathrm{i} 24$ & - & - & - & - & 0.05 & - & - & - & - & 0.50 & - & - & - & 0.70 & 0.45 & - & - \\
\hline i25 & - & - & - & 0.30 & - & 0.70 & 0.90 & 0.60 & - & - & - & 0.90 & - & 0.80 & - & - & - \\
\hline $\mathrm{i} 26$ & 1.40 & - & 0.30 & 0.70 & - & - & - & - & 1.20 & - & - & - & 1.20 & 0.55 & 0.20 & - & 0.35 \\
\hline i27 & - & 1.80 & - & - & 0.85 & - & - & 0.45 & - & - & 1.00 & 1.10 & - & - & - & 0.80 & 0.50 \\
\hline $\mathrm{i} 28$ & - & - & - & - & - & - & 1.65 & - & - & - & - & 1.05 & - & - & 1.10 & - & - \\
\hline i29 & 2.10 & - & 1.25 & - & - & 0.80 & - & - & - & 0.65 & - & - & 0.85 & - & - & - & 0.15 \\
\hline $\mathrm{i} 30$ & 1.50 & - & 0.60 & 0.75 & 0.50 & - & - & - & 0.70 & - & - & - & 1.15 & 1.30 & 0.95 & - & 0.40 \\
\hline i31 & - & 0.95 & - & - & - & - & - & - & - & - & - & 0.15 & 0.15 & - & - & 0.35 & - \\
\hline $\mathrm{i} 32$ & - & - & 0.80 & - & 0.40 & - & 0.10 & 0.20 & - & - & 0.60 & - & - & - & 1.30 & 1.00 & 0.80 \\
\hline i33 & 0.30 & 0.55 & 1.30 & - & - & 1.30 & 1.55 & - & 0.25 & 1.15 & 1.40 & 0.40 & - & - & - & 0.50 & 0.25 \\
\hline
\end{tabular}




\begin{tabular}{|c|c|c|c|c|c|c|c|c|c|c|c|c|c|c|c|c|c|}
\hline i34 & - & - & - & 1.45 & 0.80 & 0.50 & - & - & - & 0.35 & - & - & - & - & 0.75 & - & - \\
\hline i35 & - & - & - & 0.20 & - & 0.40 & 1.20 & 0.30 & - & 0.80 & - & 0.30 & - & 1.05 & - & - & 0.60 \\
\hline i36 & - & 0.25 & 1.05 & - & - & - & - & - & - & - & - & 0.85 & 0.20 & - & - & - & - \\
\hline i37 & - & - & 0.80 & - & 0.30 & 0.90 & 1.10 & 0.50 & - & 0.75 & - & - & 0.45 & - & 0.20 & - & - \\
\hline i 38 & 0.40 & - & - & 0.50 & 0.45 & - & - & - & 0.35 & 0.60 & - & - & - & 0.65 & 0.55 & - & 0.30 \\
\hline i39 & - & 0.70 & - & - & 0.65 & 0.85 & 0.80 & - & - & - & 0.70 & 0.90 & - & 0.50 & 1.05 & 0.75 & - \\
\hline $\mathrm{i} 40$ & - & - & - & 0.15 & - & - & 0.55 & 0.45 & - & 0.40 & - & - & - & 0.40 & - & - & - \\
\hline i41 & - & - & - & - & - & 0.65 & - & - & 0.85 & 0.40 & - & - & 0.35 & - & - & - & - \\
\hline $\mathrm{i} 42$ & - & - & 1.10 & - & - & - & - & - & - & - & 0.25 & - & 0.70 & - & - & 0.25 & - \\
\hline i43 & 1.00 & 0.15 & - & - & - & - & 0.30 & - & 1.60 & 0.20 & 0.50 & 0.75 & - & - & - & 0.90 & 0.60 \\
\hline i44 & - & - & - & - & 0.05 & - & - & - & - & 0.50 & - & - & - & 0.70 & 0.45 & - & - \\
\hline i45 & - & - & - & 0.30 & - & 0.70 & 0.90 & 0.60 & - & - & - & 0.90 & - & 0.80 & - & - & - \\
\hline i 46 & 1.40 & - & 0.30 & 0.70 & - & - & - & - & 1.20 & - & - & - & 1.20 & 0.55 & 0.20 & - & 0.35 \\
\hline i47 & - & 1.80 & - & - & 0.85 & - & - & 0.45 & - & - & 1.00 & 1.10 & - & - & - & 0.80 & 0.50 \\
\hline $\mathrm{i} 48$ & - & - & - & - & - & - & 1.65 & - & - & - & - & 1.05 & - & - & 1.10 & - & - \\
\hline i49 & 2.10 & - & 1.25 & - & - & 0.80 & - & - & - & 0.65 & - & - & 0.85 & - & - & - & 0.15 \\
\hline $\mathrm{i} 50$ & 1.50 & - & 0.60 & 0.75 & 0.50 & - & - & - & 0.70 & - & - & - & 1.15 & 1.30 & 0.95 & - & 0.40 \\
\hline+ & j18 & j19 & $\mathrm{j} 20$ & $\mathrm{j} 21$ & $\mathrm{j} 22$ & $\mathrm{j} 23$ & $\mathrm{j} 24$ & $\mathrm{j} 25$ & $\mathrm{j} 26$ & $\mathrm{j} 27$ & $\mathrm{j} 28$ & j29 & $\mathrm{j} 30$ & $\mathrm{j} 31$ & j32 & $\mathrm{j} 33$ & j34 \\
\hline i1 & 0.65 & - & - & - & - & - & - & - & 0.65 & - & - & 0.85 & 0.40 & - & - & 0.35 & - \\
\hline i2 & - & 0.30 & - & - & - & 1.10 & - & - & - & - & - & - & - & 0.25 & - & 0.70 & - \\
\hline i3 & - & - & - & 1.00 & 0.15 & - & - & - & - & 0.30 & - & 1.60 & 0.20 & 0.50 & 0.75 & - & - \\
\hline i4 & 0.50 & - & 0.75 & - & - & - & - & 0.05 & - & - & - & - & 0.50 & - & - & - & 0.70 \\
\hline i5 & 0.70 & 1.30 & - & - & - & - & 0.30 & - & 0.70 & 0.90 & 0.60 & - & - & - & 0.90 & - & 0.80 \\
\hline i6 & - & 0.90 & 0.80 & 1.40 & - & 0.30 & 0.70 & - & - & - & - & 1.20 & - & - & - & 1.20 & 0.55 \\
\hline i7 & - & 0.80 & 0.25 & - & 1.80 & - & - & 0.85 & - & - & 0.45 & - & - & 1.00 & 1.10 & - & - \\
\hline i8 & - & - & 0.30 & - & - & - & - & - & - & 1.65 & - & - & - & - & 1.05 & - & - \\
\hline i9 & 1.20 & - & - & 2.10 & - & 1.25 & - & - & 0.80 & - & - & - & 0.65 & - & - & 0.85 & - \\
\hline $\mathrm{i} 10$ & 1.00 & - & 1.25 & 1.50 & - & 0.60 & 0.75 & 0.50 & - & - & - & 0.70 & - & - & - & 1.15 & 1.30 \\
\hline i11 & - & 0.20 & - & - & 0.95 & - & - & - & - & - & - & - & - & - & 0.15 & 0.15 & - \\
\hline i12 & - & 0.95 & - & - & - & 0.80 & - & 0.40 & - & 0.10 & 0.20 & - & - & 0.60 & - & - & - \\
\hline i13 & - & 0.35 & - & 0.30 & 0.55 & 1.30 & - & - & 1.30 & 1.55 & - & 0.25 & 1.15 & 1.40 & 0.40 & - & - \\
\hline i14 & 0.55 & 0.50 & 0.65 & - & - & - & 1.45 & 0.80 & 0.50 & - & - & - & 0.35 & - & - & - & - \\
\hline i15 & 0.30 & - & - & - & - & - & 0.20 & - & 0.40 & 1.20 & 0.30 & - & 0.80 & - & 0.30 & - & 1.05 \\
\hline i16 & - & 0.15 & - & - & 0.25 & 1.05 & - & - & - & - & - & - & - & - & 0.85 & 0.20 & - \\
\hline i17 & 0.15 & - & 0.30 & - & - & 0.80 & - & 0.30 & 0.90 & 1.10 & 0.50 & - & 0.75 & - & - & 0.45 & - \\
\hline i18 & - & 0.60 & 0.45 & 0.40 & - & - & 0.50 & 0.45 & - & - & - & 0.35 & 0.60 & - & - & - & 0.65 \\
\hline i19 & 0.45 & - & - & - & 0.70 & - & - & 0.65 & 0.85 & 0.80 & - & - & - & 0.70 & 0.90 & - & 0.50 \\
\hline i20 & - & - & - & - & - & - & 0.15 & - & - & 0.55 & 0.45 & - & 0.40 & - & - & - & 0.40 \\
\hline i 21 & 0.65 & - & - & - & - & - & - & - & 0.65 & - & - & 0.85 & 0.40 & - & - & 0.35 & - \\
\hline $\mathrm{i} 22$ & - & 0.30 & - & - & - & 1.10 & - & - & - & - & - & - & - & 0.25 & - & 0.70 & - \\
\hline i23 & - & - & - & 1.00 & 0.15 & - & - & - & - & 0.30 & - & 1.60 & 0.20 & 0.50 & 0.75 & - & - \\
\hline i24 & 0.50 & - & 0.75 & - & - & - & - & 0.05 & - & - & - & - & 0.50 & - & - & - & 0.70 \\
\hline $\mathrm{i} 25$ & 0.70 & 1.30 & - & - & - & - & 0.30 & - & 0.70 & 0.90 & 0.60 & - & - & - & 0.90 & - & 0.80 \\
\hline
\end{tabular}




\begin{tabular}{|c|c|c|c|c|c|c|c|c|c|c|c|c|c|c|c|c|c|}
\hline i26 & - & 0.90 & 0.80 & 1.40 & - & 0.30 & 0.70 & - & - & - & - & 1.20 & - & - & - & 1.20 & 0.55 \\
\hline i27 & - & 0.80 & 0.25 & - & 1.80 & - & - & 0.85 & - & - & 0.45 & - & - & 1.00 & 1.10 & - & - \\
\hline i 28 & - & - & 0.30 & - & - & - & - & - & - & 1.65 & - & - & - & - & 1.05 & - & - \\
\hline i29 & 1.20 & - & - & 2.10 & - & 1.25 & - & - & 0.80 & - & - & - & 0.65 & - & - & 0.85 & - \\
\hline i30 & 1.00 & - & 1.25 & 1.50 & - & 0.60 & 0.75 & 0.50 & - & - & - & 0.70 & - & - & - & 1.15 & 1.30 \\
\hline i31 & - & 0.20 & - & - & 0.95 & - & - & - & - & - & - & - & - & - & 0.15 & 0.15 & - \\
\hline $\mathrm{i} 32$ & - & 0.95 & - & - & - & 0.80 & - & 0.40 & - & 0.10 & 0.20 & - & - & 0.60 & - & - & - \\
\hline i33 & - & 0.35 & - & 0.30 & 0.55 & 1.30 & - & - & 1.30 & 1.55 & - & 0.25 & 1.15 & 1.40 & 0.40 & - & - \\
\hline i34 & 0.55 & 0.50 & 0.65 & - & - & - & 1.45 & 0.80 & 0.50 & - & - & - & 0.35 & - & - & - & - \\
\hline i35 & 0.30 & - & - & - & - & - & 0.20 & - & 0.40 & 1.20 & 0.30 & - & 0.80 & - & 0.30 & - & 1.05 \\
\hline i36 & - & 0.15 & - & - & 0.25 & 1.05 & - & - & - & - & - & - & - & - & 0.85 & 0.20 & - \\
\hline i37 & 0.15 & - & 0.30 & - & - & 0.80 & - & 0.30 & 0.90 & 1.10 & 0.50 & - & 0.75 & - & - & 0.45 & - \\
\hline i38 & - & 0.60 & 0.45 & 0.40 & - & - & 0.50 & 0.45 & - & - & - & 0.35 & 0.60 & - & - & - & 0.65 \\
\hline i39 & 0.45 & - & - & - & 0.70 & - & - & 0.65 & 0.85 & 0.80 & - & - & - & 0.70 & 0.90 & - & 0.50 \\
\hline i40 & - & - & - & - & - & - & 0.15 & - & - & 0.55 & 0.45 & - & 0.40 & - & - & - & 0.40 \\
\hline i41 & 0.65 & - & - & - & - & - & - & - & 0.65 & - & - & 0.85 & 0.40 & - & - & 0.35 & - \\
\hline i42 & - & 0.30 & - & - & - & 1.10 & - & - & - & - & - & - & - & 0.25 & - & 0.70 & - \\
\hline i43 & - & - & - & 1.00 & 0.15 & - & - & - & - & 0.30 & - & 1.60 & 0.20 & 0.50 & 0.75 & - & - \\
\hline i44 & 0.50 & - & 0.75 & - & - & - & - & 0.05 & - & - & - & - & 0.50 & - & - & - & 0.70 \\
\hline i45 & 0.70 & 1.30 & - & - & - & - & 0.30 & - & 0.70 & 0.90 & 0.60 & - & - & - & 0.90 & - & 0.80 \\
\hline i46 & - & 0.90 & 0.80 & 1.40 & - & 0.30 & 0.70 & - & - & - & - & 1.20 & - & - & - & 1.20 & 0.55 \\
\hline i47 & - & 0.80 & 0.25 & - & 1.80 & - & - & 0.85 & - & - & 0.45 & - & - & 1.00 & 1.10 & - & - \\
\hline i48 & - & - & 0.30 & - & - & - & - & - & - & 1.65 & - & - & - & - & 1.05 & - & - \\
\hline i49 & 1.20 & - & - & 2.10 & - & 1.25 & - & - & 0.80 & - & - & - & 0.65 & - & - & 0.85 & - \\
\hline i50 & 1.00 & - & 1.25 & 1.50 & - & 0.60 & 0.75 & 0.50 & - & - & - & 0.70 & - & - & - & 1.15 & 1.30 \\
\hline+ & j35 & j36 & j37 & j38 & j39 & $\mathrm{j} 40$ & $\mathrm{j} 41$ & $\mathrm{j} 42$ & j43 & $\mathrm{j} 44$ & j45 & j46 & j47 & j48 & j49 & j50 & \\
\hline i1 & - & - & - & 0.65 & - & - & - & - & - & - & - & 0.65 & - & - & 0.85 & 0.40 & \\
\hline i2 & - & 0.25 & - & - & 0.30 & - & - & - & 1.10 & - & - & - & - & - & - & - & \\
\hline i3 & - & 0.90 & 0.60 & - & - & - & 1.00 & 0.15 & - & - & - & - & 0.30 & - & 1.60 & 0.20 & \\
\hline i4 & 0.45 & - & - & 0.50 & - & 0.75 & - & - & - & - & 0.05 & - & - & - & - & 0.50 & \\
\hline i5 & - & - & - & 0.70 & 1.30 & - & - & - & - & 0.30 & - & 0.70 & 0.90 & 0.60 & - & - & \\
\hline i6 & 0.20 & - & 0.35 & - & 0.90 & 0.80 & 1.40 & - & 0.30 & 0.70 & - & - & - & - & 1.20 & - & \\
\hline i7 & - & 0.80 & 0.50 & - & 0.80 & 0.25 & - & 1.80 & - & - & 0.85 & - & - & 0.45 & - & - & \\
\hline i8 & 1.10 & - & - & - & - & 0.30 & - & - & - & - & - & - & 1.65 & - & - & - & \\
\hline i9 & - & - & 0.15 & 1.20 & - & - & 2.10 & - & 1.25 & - & - & 0.80 & - & - & - & 0.65 & \\
\hline i10 & 0.95 & - & 0.40 & 1.00 & - & 1.25 & 1.50 & - & 0.60 & 0.75 & 0.50 & - & - & - & 0.70 & - & \\
\hline i11 & - & 0.35 & - & - & 0.20 & - & - & 0.95 & - & - & - & - & - & - & - & - & \\
\hline i12 & 1.30 & 1.00 & 0.80 & - & 0.95 & - & - & - & 0.80 & - & 0.40 & - & 0.10 & 0.20 & - & - & \\
\hline i13 & - & 0.50 & 0.25 & - & 0.35 & - & 0.30 & 0.55 & 1.30 & - & - & 1.30 & 1.55 & - & 0.25 & 1.15 & \\
\hline i14 & 0.75 & - & - & 0.55 & 0.50 & 0.65 & - & - & - & 1.45 & 0.80 & 0.50 & - & - & - & 0.35 & \\
\hline i15 & - & - & 0.60 & 0.30 & - & - & - & - & - & 0.20 & - & 0.40 & 1.20 & 0.30 & - & 0.80 & \\
\hline i16 & - & - & - & - & 0.15 & - & - & 0.25 & 1.05 & - & - & - & - & - & - & - & \\
\hline i17 & 0.20 & - & - & 0.15 & - & 0.30 & - & - & 0.80 & - & 0.30 & 0.90 & 1.10 & 0.50 & - & 0.75 & \\
\hline
\end{tabular}




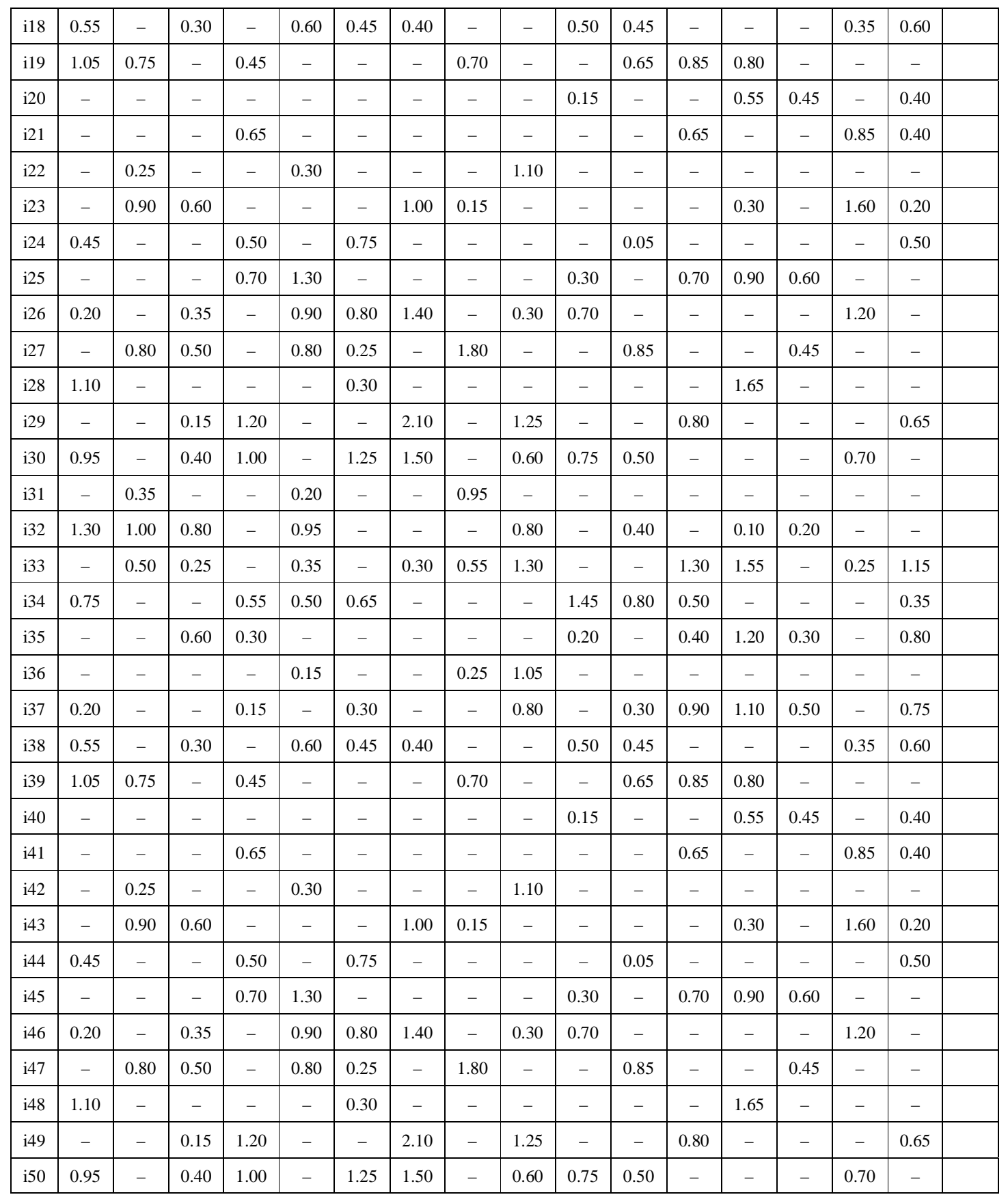

\section{Results $T C$ and $T F$ minimization by ARS}




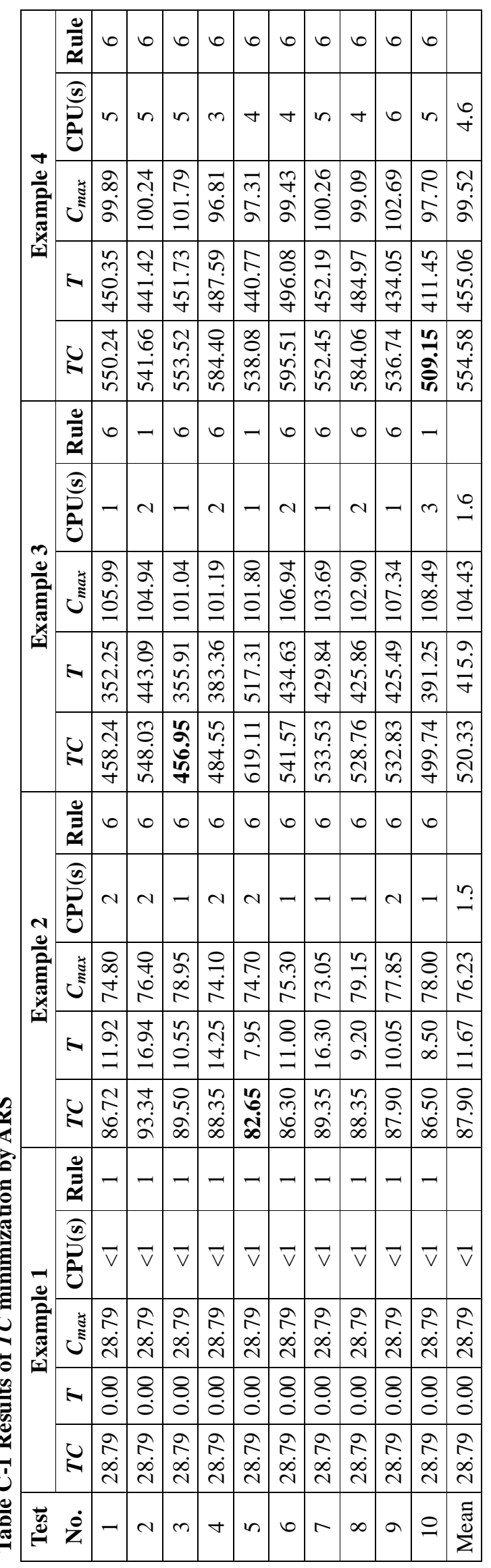

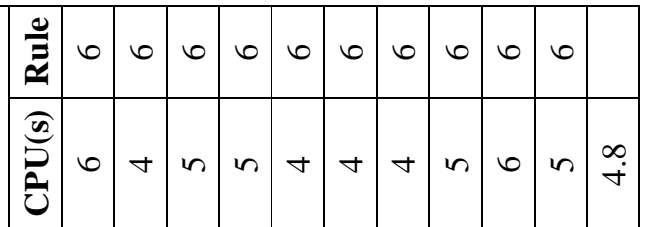

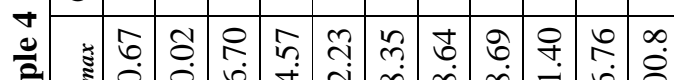
ปै 되

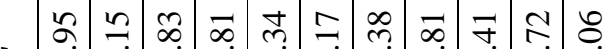

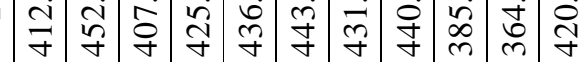

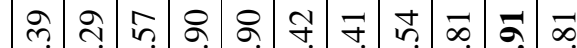

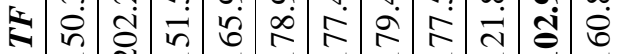
正 $\cong$ 三 三 三 三

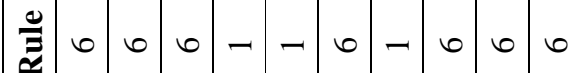

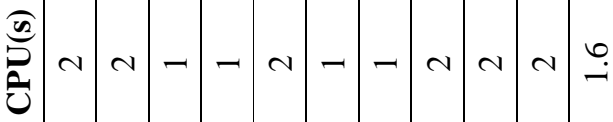

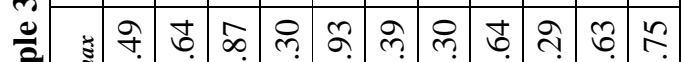
盯 å

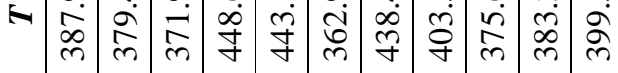
๔ ஒ

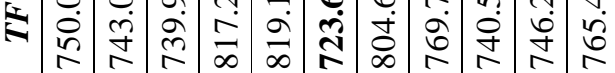

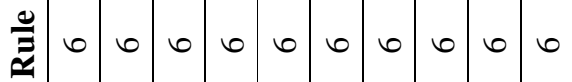

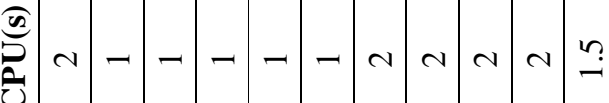

产

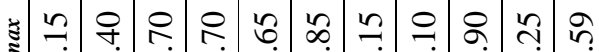
viล $\checkmark$ œ

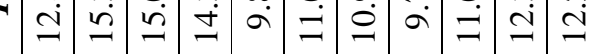
×

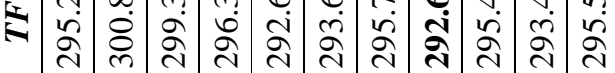

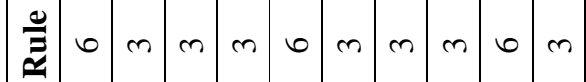

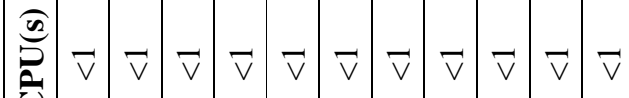
它

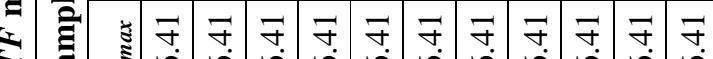

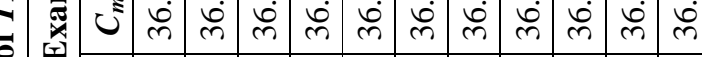
泀

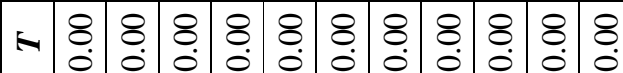

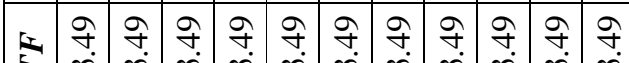
- $\dot{r} \ddot{r} \ddot{r} \dot{r} \ddot{r} \dot{r} \ddot{r}$

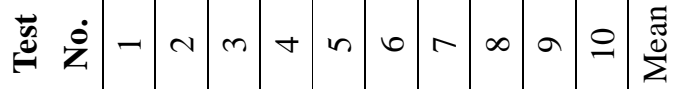

